\title{
A Pilot Study with Spanish-Speaking Latina Survivors of Domestic Violence Comparing EMDR \& TF-CBT Group Interventions
}

\author{
Helen Harris' ${ }^{1}$, Viviana Urdaneta², Viviana Triana ${ }^{3}$, Charity Samantha Vo ${ }^{4}$, \\ Destinee Walden ${ }^{5}$, Dennis Myers ${ }^{1}$ \\ ${ }^{1}$ Baylor University, Waco, TX, USA \\ ${ }^{2}$ Private Practice, Oakland, CA, USA \\ ${ }^{3}$ The Pastoral Counseling Center, Dallas, TX, USA \\ ${ }^{4}$ Middle Tennessee State University, Murfreesboro, TN, USA \\ ${ }^{5}$ Community Options for Families and Youth, Pleasant Hill, CA, USA \\ Email: Helen_Harris@baylor.edu, vivianasemdr@gmail.com,csvo@mtsu.edu,Destineewalden.sw@gmail.com, \\ Dennis_Myers@baylor.edu
}

How to cite this paper: Harris, H., Urdaneta, V., Triana, V., Vo, C.S., Walden, D. and Myers, D. (2018) A Pilot Study with Spanish-Speaking Latina Survivors of Domestic Violence Comparing EMDR \& TFCBT Group Interventions. Open Journal of Social Sciences, 6, 203-222.

https://doi.org/10.4236/jss.2018.611015

Received: October 20, 2018

Accepted: November 19, 2018

Published: November 22, 2018

Copyright (c) 2018 by authors and Scientific Research Publishing Inc. This work is licensed under the Creative Commons Attribution International License (CC BY 4.0)

http://creativecommons.org/licenses/by/4.0/

\begin{abstract}
This pilot study compared two evidence-informed group treatment methods with 19 Spanish-speaking Latina survivors of domestic violence. Spanishspeaking therapists trained in Eye Movement Desensitization Reprocessing (EMDR) and Trauma-Focused Cognitive Behavior Therapy (TFCBT) led the groups. Assessments of Post-Traumatic Stress Disorder (PTSD) in participants using the SPRINT instrument taken after psychoeducational preparation/prior to trauma processing, and at the end of treatment demonstrated improvement in participants in the EMDR groups and in the TFCBT groups with more reduction of PTSD symptoms in the EMDR groups, although the findings are limited by small sample size and so are not generalizable. The results suggest that there is a need for additional study both on the effectiveness of group treatment for this population and on the best evidence-based approaches.
\end{abstract}

Keywords

PTSD, Survivors, Sexual Trauma, Latinas, EMDR, TFCBT

\section{Introduction}

Latinas may be at increased risk of intimate partner violence (IPV) particularly when isolated by immigration status, language barriers, and low socioeconomic 
status; this increases risk for Post Traumatic Stress Disorder (PTSD). In cases of survivors with limited access to individual evidence-based treatments for PTSD, therapy is expensive and limited, with long waiting lists. This study was initiated by therapists in a Texas domestic violence program who were concerned about long waiting lists of Spanish-speaking Latina survivors of domestic violence. The agency had few Spanish-speaking therapists with limited availability for individual therapy schedules. The therapists explored offering group therapy to Spanish-speaking survivors on the waiting list and committed to evaluating their group treatment for PTSD. The therapists requested research faculty at their graduate social work program for assistance. This resulted in a pilot study addressing group treatment modalities for effectiveness in treating PTSD symptoms in Latina survivors of IPV. The Spanish speaking therapists were trained in Eye Movement Desensitization Reprocessing (EMDR) and Trauma-Focused Cognitive Behavior Therapy (TF-CBT), each of which was delivered in group format.

\section{Literature Review}

There are several areas of pertinent literature for this study. This literature review includes interpersonal violence with a particular focus on Latina survivors of IVP and barriers to help seeking for this population; Additionally, the literature review covered PTSD after IPV; evidence-based treatment for PTSD, use of group interventions, and both TFCBT and EMDR delivered in group format.

IPV is a pervasive social problem that, according to global statistics, affects one in three women in their lifetime [1]. From 2005-2012, 22\% of all violent crime committed on human beings in the United States was domestic violence (DV) [2]. Fitz-Gibbon, Walklate, McCulloch, and Maher define IPV as "abusive behavior by a person within an intimate relationship including current or past marriages, domestic partnerships, or de facto relationships" [3]. According to Regan \& Durvasula [4], personal, interpersonal or relational, and environmental factors compose three broad categories of risk factors for experiencing IPV. Factors that affect the risk of IPV include socioeconomic status, prior IPV exposure and childhood abuse, economic and living conditions, existing political climate, and sociocultural norms or customs; conflict resolution strategies and relationship status [4]. Alvarez, Davidson, Fleming, and Glass [5] identified additional risk factors to IPV within the Latino population including immigration status, machismo (cultural belief that malesshould dominate relationships), and acculturation stress.

Latinos, persons of Hispanic descent, are one of the most rapidly growing populations in the United States [5] comprising about $16 \%$ of the population [6]. Thirty-seven percent of Hispanic women in the United States report being the victim of IPV in their lifetime [7]. Latina women are more likely to experience more severe adverse effects from IPV than are non-Latina white women [5] though when variables such as age, alcohol use, location, and family history are controlled, these differences disappear [8]. Since some Latina women are not 
able to access evidence-based interventions for IPV, the authors stressed the importance of developing interventions that are culturally and linguistically appropriate to empower Latinas to overcome barriers to seeking help [5]. In this article, IPV means both interpersonal violence and domestic violence, and the terms Hispanic and Latina are used interchangeably.

Chapman and Monk [1] asserted that shame, denial, and guilt are common factors that act as help seeking barriers for victims of IPV. Additional barriers to help seeking identified in the literature include damaged self-esteem, learned helplessness, fear for safety, fear of involvement of child welfare services, economic dependence on perpetrator, and emotional investment in the relationship [1]. For some Latinas, resistance to help seeking may be related to a fear of deportation for undocumented immigrants, either themselves or their partners, and a desire to stay in the marriage due to traditional norms. Other factors might include language barriers and lack of understanding of the social, legal, and health systems [5]. They stated that "Latinas (especially monolingual Spanish speakers) are more likely to negate the severity of abuse, less likely to use formal services to address IPV, and when they do eventually use formal services, it is after extended periods of experiencing abuse" (pp. 2-3).

\section{PTSD}

According to the World Health Organization [9], IPV affects women's physical and mental health through direct pathways, such as injury, and indirect pathways, including prolonged stress resulting in PTSD symptoms [9]. The trauma of abuse and violence can result in post-traumatic stress including anxiety, depression, low self-esteem, and relational difficulties [1] [4] [10]. PTSD, commonly experienced by those who have suffered IPV, may include avoidance responses, arousal, re-experiencing of the trauma, dissociation, negative cognitions, and mood [9] [11]. Another defining feature of PTSD is that the storage of memories related to the traumatic event is fragmented and sometimes inhibited [9]. The brain's normal information processing system is often interrupted after a traumatic event requiring intervention.

The literature on providing culturally competent mental health services to Hispanic populations suggests that a key variable is access to treatment [12]. Prelli [13] found that group-based therapeutic interventions may be particularly valuable for victims of domestic violence, particularly where there are limited resources and high costs. Stacciarini, O'Keefe, and Mathews [14] found group therapy with Latina women to be a cost-effective approach. Some benefits of group sessions include feelings of empowerment, improvements in self-awareness, peer support, normalizing symptoms of PTSD, and maximizing staff resources that are often limited [15] [16].

Empirically supported treatments for trauma include Trauma Focused Cognitive Behavioral Therapy (TF-CBT) and Eye Movement Desensitization Reprocessing (EMDR) therapy [17] [18] [19] [20]. Re-experiencing the traumatic event is the primary focus in many treatments of PTSD with a goal of reducing 
intrusive images, thoughts, and nightmares related to the trauma. Other important treatment approaches include relaxation techniques; providing a safe environment to facilitate the expression of emotions; and finding meaning to re-establish a sense of control and predictability [11]. The Spanish speaking therapists in the agency and in this study were both trained in TF-CBT and in EMDR.

TF-CBT is based on learning and cognitive theories and addresses distorted beliefs and attributions related to the trauma. TF-CBT includes a supportive environment in which survivors are encouraged to talk about their traumatic experiences and to learn new skills to help process thoughts and feelings related to traumatic life experiences [21]. Psychoeducation, imaginal and in vivo exposure, relaxation training, homework exercises, cognitive restructuring, and discussion around social support comprise the common areas of TF-CBT [22]. The literature suggests that cognitive processing of the meaning of the trauma and exposure to the traumatic memory are the active ingredients of TF-CBT [23].

A limitation of TF-CBT is that most of the evidence is in work with children and their parents in families with trauma experience. The exposure elements can be initially draining but ultimately beneficial [24]. The California EvidenceBased Clearinghouse (CEBC) [25] classifies TF-CBT as a level 1 intervention, which is an intervention that has, strong empirical support [25]. The Agency for Healthcare Research and Quality (AHCPRQ) [26] classifies TF-CBT as a level A intervention (highest empirical support).

TF-CBT group protocol is very similar to the individual implementation of TF-CBT [27]. Studies support the efficacy of TF-CBT group delivery particularly with young children who are sexual abuse survivors in the United States [28] [29]. There are also studies with war-affected adolescents in the Democratic Republic of Congo (DRC) [30] and one trial with orphaned children in Tanzania [15]. Deblinger, Stauffer, and Steer [28] found the PTSD symptoms did not significantly change, impacted perhaps by the young age of the participants. However, in the DRC studies, PTSD symptoms were significantly decreased [27].

EMDR therapy is based on the adaptive information processing (AIP) model and consists of eight phases with standardized protocols [31]. The AIP model posits that memory networks holding the experiences of trauma must be processed in order to connect to other networks that hold information of adaptive nature. In several quantitative meta-analyses, Acarturk et al. [32] and Chen et al. [33] found that the studied disorders and symptoms were significantly reduced with effect sizes that were moderate to large, supporting EMDR as an optimal psychotherapy for patients with PTSD. However, none of the studies included Latinas and there were often small sample sizes. In 2013, deBont, van Minnen, and de Jongh found both PE and EMDR to be effective in treating PTSD [34]. Ringel [35] addressed specifically EMDR effectiveness with adult survivors of sexual abuse. The World Health Organization guidelines list EMDR as an effective treatment for PTSD [9].

There currently is a lack of literature addressing the use of EMDR with Latina 
women who are survivors of IPV. However, EMDR is ranked by the Agency for Health Care Policy and Research (AHCPR) [26] at a Level A in regards to adult populations, the highest level of empirical support.

There are two protocols for doing EMDR in group (EMDR-IGTP and G-TEP). The Group Traumatic Episode Protocol (G-TEP) was developed by Elan Shapiro to construct a group protocol that contains the power of EMDR and the AIP model and closely resembles the individual protocol. The G-TEP is an adaptation of the Recent-Traumatic Event Protocol (R-TEP) for use with groups of adults, older children, and adolescents who have had recent traumatic experiences or longer term life-changing events with on-going consequences [36]. Jarero and Artiga [37] developed an EMDR group therapy protocol (EMDRIGTP) in response to the high number of requests for mental health care following hurricane Pauline in Mexico in 1997 [38]. EMDR-IGTP has been used with children and adults in different places of the world. Several studies report its effectiveness with children and adults in response to disasters, ongoing war trauma, ongoing geopolitical crisis, war refugee displacement, work accidents, and severe IPV [39]. The therapists used this EMDR protocol in their study because of their familiarity with it and its effectiveness.

\section{Comparison of EMDR and TFCBT}

McGuire, Lee, and Drummond [9] compared TF-CBT and EMDR. Similarities included information-processing theories; focus on the traumatic event in order to understand personal meanings and consequences of the event; activation of the fear memory network by presenting information matching elements of the fear structure and introducing corrective information that is not compatible with these elements; adhering to theories of fear structures; and introduction of corrective information.

Differences between EMDR and TF-CBT include the use of imaginal exposure (flooding approach) in TF-CBT requiring the individual to vividly relive the traumatic experience. EMDR however "progresses through chains of associations that utilize imaginal exposure in short bursts" [31]. TF-CBT requires significant therapist direction and homework while EMDR relies on the processing of the client's brain with less narrative interaction required in group.

While the literature on TF-CBT and EMDR with the Latina population is limited, the literature on both modalities includes several population groups in several countries with generally consistent results. Group treatment provides the possibility of PTSD treatment that can reduce wait times and reach a population that is often marginalized.

\section{Methodology}

This study compared treatment outcomes of survivors of IPV in purposive strategically assigned EMDR and TF-CBT treatment groups. It was conducted in a domestic violence shelter and treatment program in Texas with a waiting list of 
Spanish-speaking Latina survivors. The study was approved by the Institutional Review Board (IRB) of Baylor University. Informed consent was obtained from all participants before enrollment. The study is a quasi-experiment with internal validity bolstered by treatment modality between TF-CBT and EMDR driven by client schedule. Groups were held on different days and assignment of participants to groups was based on their schedule availability rather than on assignment for specific modality.

\section{Research Questions}

This study of Spanish-speaking Latina clients who are survivors of domestic violence focused on the following questions:

1) How effective are TF-CBT and EMDR group treatment for addressing PTSD symptoms as measured by the Short PTSD Rating Interview (SPRINT) instrument?

2) Is EMDR group therapy more effective than TF-CBT group therapy in reducing PTSD symptoms?

\section{Design}

The study is a two-group comparison design conducted at a non-profit women's shelter providing safety, counseling, and professional therapy services to female victims of IPV and their children in both a residential and an outpatient location. At the time of the study, the agency held on average 12 weekly groups in Spanish for women survivors of IPV with a consistent wait list for counseling services of as many as 100 Spanish-speaking Latinas. At the time of the study, wait list times averaged $3-6$ months for therapy services. Two of the researchers were Spanish-speaking therapists who conducted the groups and collected data at the agency including de-identifying all data prior to analysis.

Women included in the sample completed an intake and initial baseline evaluation for PTSD prior to the study to be included on the waitlist. These clients were allowed the opportunity to see an individual therapist or caseworker briefly while waiting for a therapy opening in individual or group treatment. Spanish-speaking therapists at the non-profit were equipped to provide evidencebased therapy for survivors of IPV with symptoms of PTSD, including EMDR and TF-CBT therapies.

\section{Participants and Treatment Assignment}

The study recruited participants through convenience sampling. Inclusion criteria included the following: adult, female, Latina, Spanish speaking, survivor of IPV, and waiting for a therapy opening in the agency. Participants also had to be available and able to attend treatment groups at the times provided. Exclusion criteria included gender, language, severe intellectual impairment, and lack of transportation to the treatment group. Males were excluded from the group because the organization provides services only to women and children. Non-Spanish 
speaking clients were excluded from the group because the groups were conducted in Spanish.

Participants on the waiting list were contacted by phone by one of the researcher therapists and were offered group treatment at the agency. Thirty-four participants expressed willingness to participate in one of the groups. Options included groups on Monday through Thursday afternoons. Participants identified a day or days that they were able to attend the group. Participants were placed into groups through purposive strategic assignment, with the limitation of only including women in each group's assignment who could attend on that weekly treatment day. Women who reported availability on only one day were automatically assigned to that group whereas those who reported availability on two or more days were assigned strategically to any of their options by writing separately their days of availability on a piece of paper, putting them in a bag, and one of the researchers picked a piece of paper with the day of the week for the group to which that the woman was assigned. Group size was limited to a maximum of 10 participants per group, based on agency parameters, with an average membership of 6 - 8, depending on availability.

Once the group composition was completed, each group had an equal opportunity for assignment to one of the two treatments. The assignment procedure involved the researcher writing EMDR on two pieces of paper and TF-CBT on two, putting them into a bag, blindly drawing a treatment option name from the bag, and linking it with a treatment group on a particular the day of the week, beginning with Monday and continuing through Tuesday, Wednesday, and Thursday. Four groups were completed in the summer. The researchers followed the same process for four additional fall groups including those who had been added to the waiting list during the fall.

Group facilitators discussed and secured informed consent from participants. Those who elected not to participate in a research group were provided with alternative therapy options. Over the course of the treatment, eight participants dropped out of the summer groups and six participants dropped out of the fall groups due to transportation difficulties, job, and scheduling conflicts. These participants were referred to alternative group or individual treatment. Nineteen participants completed the study in full. The flow of participants from waitlist through treatment is shown in Figure 1.

\section{Measures}

For this study, PTSD symptoms were measured using the SPRINT scale, a scale routinely used by the agency to assess for PTSD symptoms. Permission to use this scale was obtained by the researchers through written communication with the developer, Jonathan R. T. Davidson. The SPRINT instrument can be viewed by contacting the developer or by referencing the study by Connor and Davidson [40]. Vaishnavi, Payne, Connor, and Davidson [36] found SPRINT to be empirically valid and comparable to the Clinical Administered PTSD Scales for 


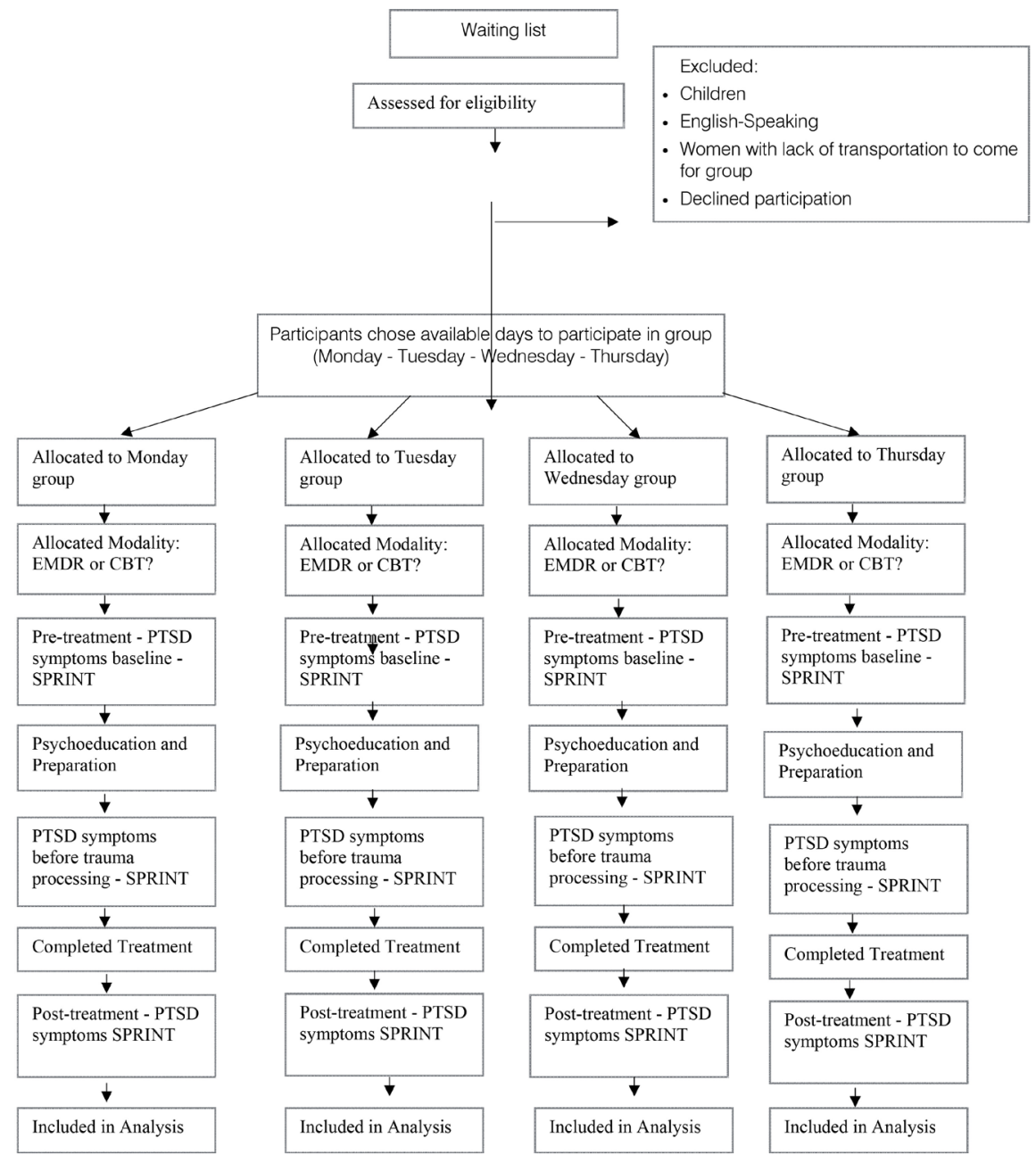

Figure 1. Flow of participants.

DSM. The authors found comparable reliable results with shorter testing sessions. For purposes of the study, participants completed the SPRINT at the beginning of the group experience, at the beginning of the trauma processing of TF-CBT or EMDR intervention group sessions, and at the end of the group experience.

The SPRINT is an 8-item assessment that measures PTSD core symptoms including somatic malaise, functional impairment, and stress vulnerability [40] Connor and Davidson [40] administered the SPRINT in a clinical trial of PTSD with a population survey that assessed the prevalence of PTSD. Validity of SPRINT was "assessed against the MINI structured interview, the Davidson Trauma Scale, Treatment Outcome for PTSD Scale, Connor-Davidson Resilience Scale, Sheehan Stress Vulnerability Scale, Sheehan Disability Scale and Clinical Global Impressions of Severity and Improvement Scales" (p. 279). The authors found good test-retest reliability (ICC $=0.778)$, convergent $(r=0.073)$ and divergent validity $(r=0.10)$, and internal consistency ( $\alpha=0.77$ at baseline and $\alpha=0.88$ at endpoint). Evidence supported the responsiveness of SPRINT to changes in symptoms over time and a correlation with comparable PTSD symp- 
tom measures. Ninety-six percent diagnostic accuracy was associated with a score of 14 - 17 in trauma victims; highest efficiency, for those with PTSD, corresponded to a range of 11 - 13. Connor and Davidson [40] found that solid psychometric properties were demonstrated by SPRINT and that this assessment can be utilized as a valid, reliable, and homogenous measure of illness severity of PTSD and of global improvement. In a separate study, Leiva-Bianchi and Gallardo [41] reported $\alpha=0.916$ for the reliability of SPRINT.

\section{Treatment Procedures}

This study took place in a 12-month period from June 2015 to June 2016 at the outpatient facility of agency. The research included a total of four EMDR groups and four TF-CBT groups (four 8 - 12 week summer groups and four 8 - 12 week fall groups for a total of four EMDR groups and four TF-CBT groups). Groups met an average of 10 times each. Each group started with 8 - 10 participants led by Spanish-speaking licensed therapy staff of the agency. Two summer and two fall groups were TF-CBT groups; two summer and two fall groups were EMDR groups.

The group facilitators were trained in both modalities. In attempt to eliminate participant selection bias, participants and group facilitators were initially blind to their assigned treatment modality. Each group consisted of the following format: Sessions 1 - 5 focused on psychoeducation and safety planning, resource development, and preparation for trauma processing. Sessions 6 - 9 focused on trauma processing and desensitization using either EMDR or TF-CBT. The last session focused on closure and coping skills. In order to assure fidelity to the curriculum, facilitators followed the session descriptions in Figure 2. TF-CBT sessions used the TF-CBT manual. Facilitators were also supervised by two of the researchers. The work of each of the sessions for each treatment model is shown in Figure 2.

\section{Data Management and Privacy/Confidentiality}

All participant names were replaced by a code number. All SPRINT instruments were coded with the participants' code number and stored securely in the agency. All data were collected in hard-copy format by researchers at the agency and kept in a locked file cabinet. Hard copies were scanned into a. PDF file and emailed to the university researchers for analysis using a password-protected, encrypted computer for analysis. Emails were deleted from the server and the computer files. Data entry was spot-checked to ensure accuracy.

\section{Data Analysis}

The researchers performed a two-way mixed ANOVA to assess the main effects for treatment type and passage of time as well as possible interaction using SPSS 25. Treatment type (i.e., TF-CBT, EMDR) served as the between-subjects factor. Time served as our within-subjects factor with three different points of observation: pre-intervention, mid-intervention (before trauma processing), and 


\begin{tabular}{|c|c|}
\hline EMDR & TF-CBT \\
\hline $\begin{array}{ll}\text { Session 1: } \\
- & \text { SPRINT Scale } \\
- & \text { Ice breaker } \\
- & \text { Expectations and guidelines } \\
- & \text { What to do if a crisis happened between sessions? } \\
- & \text { Psychoeducation around EMDR and BLS } \\
& \text { Containment - To go boxes and worry dolls } \\
\end{array}$ & $\begin{array}{ll}\text { Session 1: } \\
-\quad \text { SPRINT Scale } \\
- & \text { Ice breaker } \\
- & \text { Expectations and guidelines } \\
- & \text { What to do if a crisis happened between sessions? } \\
- & \text { Psychoeducation around TF-CBT } \\
- & \text { Homework: Write positive thoughts during the week or things that make them feel well } \\
\end{array}$ \\
\hline $\begin{array}{l}\text { Session 2: } \\
-\quad \text { Pleasant memory from the week } \\
\text { - Psychoeducation about Domestic violence - types of abuse, cycle of violence, and common } \\
\text { feelings and responses } \\
\text { - }\end{array}$ & $\begin{array}{ll}\text { Session 2: } \\
-\quad \text { Pleasant memory from the week } \\
-\quad \text { Psychoeducation about Domestic violence - types of abuse, cycle of violence, and common feelings and } \\
\text { responses } \\
-\quad \text { Psychoeducation about trauma. Following manual for TF-CBT } \\
-\quad \text { Abdominal breathing } \\
\text { Homework: Practice abdominal breathing }\end{array}$ \\
\hline 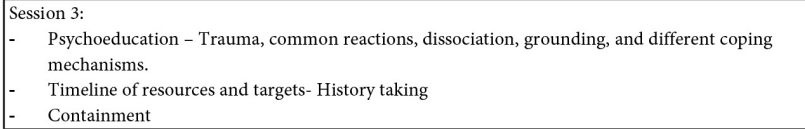 & $\begin{array}{l}\text { Session3: } \\
-\quad \text { What does it mean to feel relaxed. Follow manual TF-CBT } \\
-\quad \text { Practice relaxation } \\
-\quad \text { Safe Place } \\
\text { Homework: Think about your safe place when feeling stressed. }\end{array}$ \\
\hline $\begin{array}{l}\text { Session 4: } \\
-\quad \text { Issue driven treatment planning } \\
-\quad \text { Safe place BLS }\end{array}$ & $\begin{array}{l}\text { Session 4: Feelings } \\
-\quad \text { Psychoeducation about feelings } \\
-\quad \text { Feelings in the body. How intense feelings are. Toolbox to cope with uncomfortable feelings. } \\
\text { Homework: Recognize feelings during the week. Fill out the log of feelings in the TF-CBT. }\end{array}$ \\
\hline $\begin{array}{l}\text { Session 5: } \\
\text { - Resource development: Nurturing figure, wisdom figure, circle of support, and RDI - Mastery, } \\
\text { relational and symbolic resources }\end{array}$ & $\begin{array}{ll}\text { Session } 5: \text { Thoughts and actions } \\
-\quad \text { Stop your feelings } \\
-\quad \text { Difference between feelings, thoughts, and behaviors } \\
-\quad \text { Problematic thoughts } \\
\text { Homework: Take note of your thoughts }\end{array}$ \\
\hline 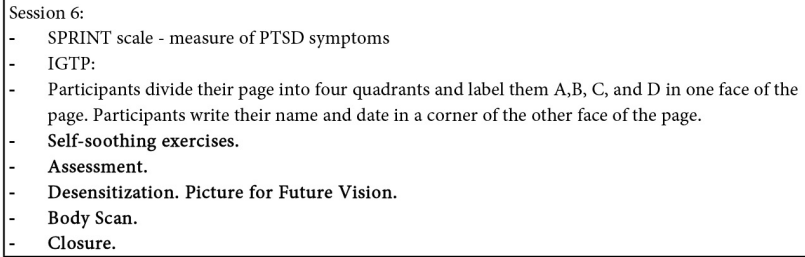 & $\begin{array}{l}\text { Session 6:Writing your story } \\
-\quad \text { SPRINT scale - Measure of PTSD symptoms } \\
\text { - Time for writing their story: First scene: before the event, Second scene: Describe the event(s) that } \\
\text { cause you disturbance, Third scene: After the event(s) } \\
\text { - Remind them about the toolbox from session four to cope with uncomfortable feelings. } \\
\text { Homework: Utilize the toolbox. }\end{array}$ \\
\hline 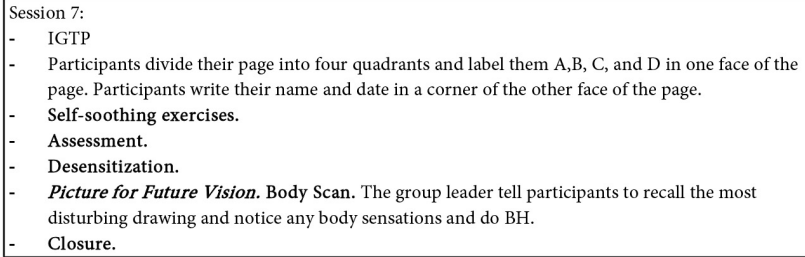 & $\begin{array}{l}\text { Session 7: Telling your story } \\
-\quad \text { Participants told their story answering questions (per TF-CBT manual). Therapists help to identify } \\
\text { thoughts, feelings, behaviors and to reframe it. } \\
\text { - The questions are: } \\
\text { - } \quad \text { Why did this happen to me? } \\
-\quad \text { Who is responsible for the upsetting/confusing event(s)? } \\
-\quad \text { How will the upsetting/confusing event(s) affect me in the future? } \\
\text { - } \quad \text { Sow have the upsetting/confusing events affected my family? }\end{array}$ \\
\hline 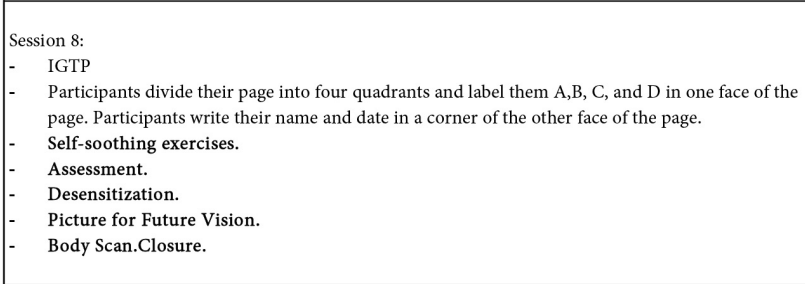 & $\begin{array}{l}\text { Session 8: Telling your story } \\
-\quad \text { Participants told their story answering questions (per TF-CBT manual). Therapists help to identify } \\
\text { thoughts, feelings, behaviors and to reframe it. } \\
\text { - The questions are: } \\
\text { - } \\
\text { - Since the event(s), my view of myself has changed in these ways: } \\
\text { - } \quad \text { Coming to therapy has changed me and my family in these ways: } \\
-\quad \text { If I had a friend that went through a similar upsetting/confusing event, I would give him or her this } \\
\text { advice: } \\
\text { If my friend thought that talking about the upsetting/confusing event would be too hard, I would tell } \\
\text { him or her: }\end{array}$ \\
\hline 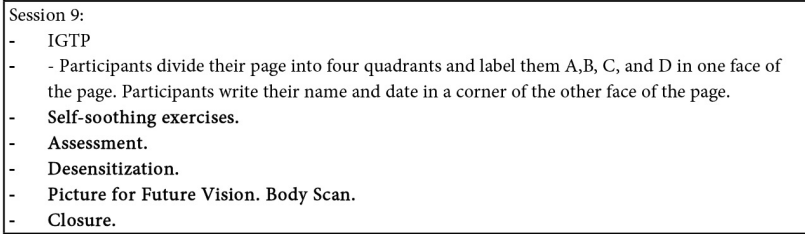 & $\begin{array}{l}\text { Session 9: Telling your story } \\
-\quad \text { Struggling with memories from trauma } \\
-\quad \text { Maintaining safety - physical and emotional }\end{array}$ \\
\hline $\begin{array}{l}\text { Session 10: } \\
-\quad \text { Future template } \\
-\quad \text { SPRINT scale to measure PTSD symptoms } \\
-\quad \text { Certificates of competition and celebration }\end{array}$ & $\begin{array}{ll}\text { Session 10: Closing and Farewell } \\
- & \text { Circle of internal and external resources } \\
- & \text { Fabulous future } \\
- & \text { SPRINT scale to measure PTSD symptoms } \\
- & \text { Certificates of competition and celebration }\end{array}$ \\
\hline
\end{tabular}

Figure 2. Group sessions.

post-intervention. The dependent variable was trauma symptomology as measured on the SPRINT scale with scores ranging from 0 to 32). The researchers screened for missing data and opted to exclude all participants from analyses missing pre-intervention and/or post-intervention scores. We conducted Tukey post-hoc analyses for all statistically significant main effects. We retrospectively calculated 
our achieved power using $\mathrm{G}^{\star}$ Power.

\section{Findings}

The study initially included 34 participants who are Latina, Spanish-speaking women and survivors of IPV. This sample was significantly smaller than the 80 plus anticipated participants, significantly limiting any generalizability of the study. Many on the waiting list were unable to commit to weekly group participation at the scheduled group times or decided they would rather wait for individual treatment appointments. This is consistent with the literature review about difficulty accessing services for Latina clients. By the completion of the study, 14 participants dropped out, leaving only 20 participants, 19 for whom the researchers had all observations. In the beginning of the study, 14 participants were in the EMDR group; 8 completed the study (57\%). The TF-CBT group consisted of 20 participants at the beginning of the study; 12 completed the study (60\%). The difference in numbers beginning in the two groups was due to the unforeseen circumstance of more participants being able to attend on the weekday meeting time of the TF-CBT group. Drop-out reasons were the same for both groups including financial, i.e., securing employment and needing to work, or lack of transportation to group. Ages of participants ranged from 21 years old to 60 years old, with the median age of participants being 42.5 .

\section{Participant Domestic Violence Experience}

As described in Table 1, the two groups of women who completed the study had very similar rates of experience for the various forms of IPV. While the EMDR group experienced a higher rate of physical abuse, sexual abuse, and abuse as a child, the TF-CBT group had a higher rate of childhood experiences with IPV. All of the participants in both groups had experienced verbal/emotional abuse. The percentages of participants who experienced abuse are shown in Table 1.

\section{Sprint Score (PTSD) Analysis}

The data reasonably conformed to the assumptions of a mixed ANOVA. There were no significant outliers in any cell as evidenced by examining studentized residuals. Visual inspection of Q-Q plots revealed normally distributed SPRINT scores for each cell. Additionally, the data conformed to the assumptions of homogeneity of variances (as assessed by Levene's test of homogeneity of variance, $p>0.05$ ), homogeneity of covariances (as assessed by Box's test of equality of covariance matrices, $p>0.05$ ), and sphericity (as assessed by Mauchly's test of sphericity, $p>0.05$ ).

There was no statistically significant interaction between treatment and time, $F(2,34)=2.194, p>0.05$, partial $\eta^{2}=0.114$. The main effect for treatment type was not statistically significant, $F(1,17)=2.428, p>0.05$, partial $\eta^{2}=0.125$. The main effect for time was statistically significant, $F(2,34)=3.686, p<0.05$, partial 
Table 1. Percentage of group members who have experienced various forms of abuse.

\begin{tabular}{cccc}
\hline & $\operatorname{EMDR}(\mathrm{n}=7)$ & TF-CBT $(\mathrm{n}=12)$ & Combined $(\mathrm{n}=19)$ \\
\hline Verbal/emotional abuse & 100 & 100 & 100 \\
Physical abuse & 100 & 83.3 & 91.65 \\
Sexual abuse & 71.4 & 58.3 & 64.85 \\
Abuse as a child & 57.1 & 41.7 & 49.4 \\
Witness to domestic violence as a child & 42.9 & 66.7 & 49.4 \\
\hline
\end{tabular}

$\eta^{2}=0.178$. We ran all possible pairwise comparisons for the main effect of time. Tukey post hoc tests revealed a statistically significant difference between pre-treatment and post-treatment scores. More specifically, pre-treatment SPRINT scores were on average 3.435 points, 95\% CI [0.401, 6.468] higher than posttreatment SPRINT scores, $p<0.05$. The mean Sprint scores from pre to post test by treatment group are shown in Table 2 .

As illustrated in Table 2, the EMDR group showed the greatest improvement in PTSD symptomology based on pre- and post-treatment SPRINT scores. There are three data points depicted in Figure 1 due to the SPRINT being administered in the middle of treatment along with pre- and post-testing. The TF-CBT group experienced an increase in SPRINT scores between the midway point and the post-test, which coincided with the trauma narrative portion of treatment. The results show that the EMDR group experienced a slightly greater reduction in PTSD symptomology based on the SPRINT scores. The graph of Sprint scores by group is shown in Figure 3.

\section{Summary of Findings}

The generalizability of the findings of this study are impacted by the much smaller sample of participants than anticipated. Both TF-CBT and EMDR in group format were supported as clinically significant, albeit not statistically significant, in reducing PTSD symptomology in this study with this particular population. All effect sizes are medium to large, yet only the main effect for time is statistically significant due to low power (0.42). Although it is not possible to draw any firm conclusions due to the low power, EMDR group therapy showed to be slightly more effective than TF-CBT in this study. The TF-CBT participants reported higher symptomology for PTSD on the post-test SPRINT than the EMDR group. In Figure 1, the TF-CBT group's scores begin to go down, but then they slightly increase after the trauma narrative portion of treatment. EMDR, however, has a continuous decrease in SPRINT scores regardless of point in the study.

One of the findings from this research is that the psychoeducation and preparation procedures were helpful for both groups while the trauma processing with EMDR-IGTP was more effective to reduce symptoms of PTSD than the TF-CBT trauma narrative approach. This suggests than trauma stabilization and self- 
Table 2. SPRINT mean scores and standard deviations by group.

\begin{tabular}{ccc}
\hline & EMDR $(n=7)$ & TF-CBT $(n=12)$ \\
\hline Pre-test & $19.14(3.63)$ & $22.25(7.34)$ \\
Previous to trauma processing & $17.86(4.60)$ & $19.50(7.55)$ \\
Post-test & $13.86(4.78)$ & $20.67(5.25)$ \\
\hline
\end{tabular}

Note. Standard deviations are denoted in parentheses.

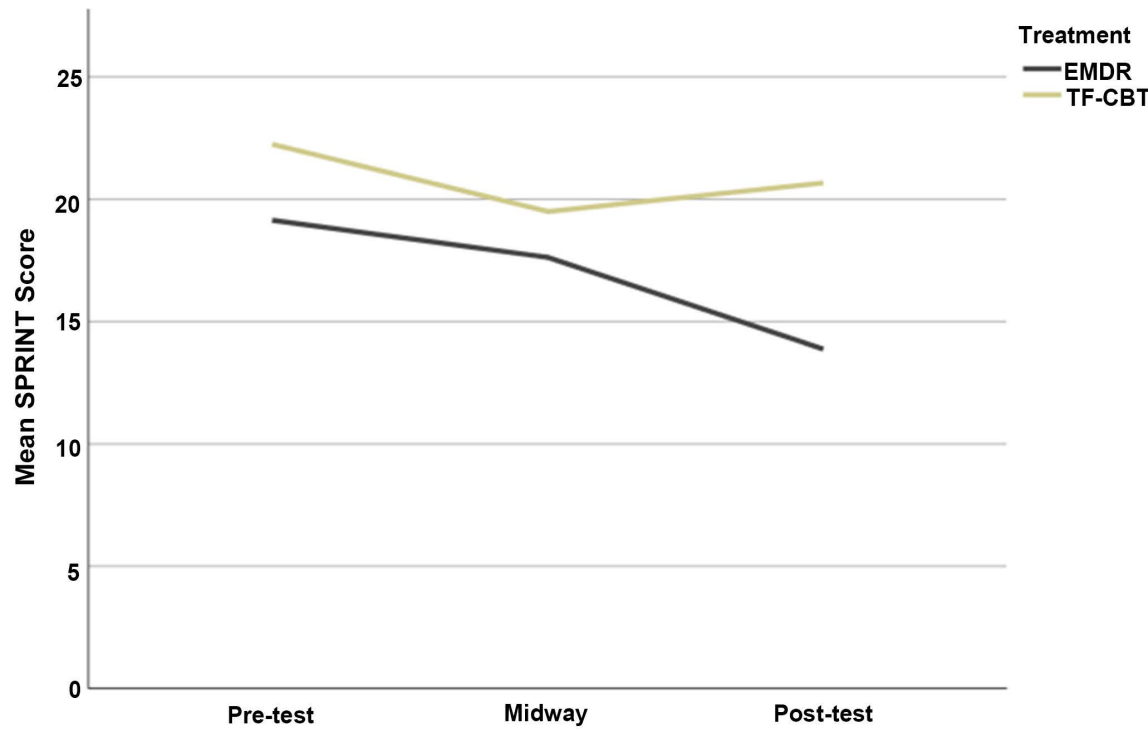

Figure 3. Mean sprint scores.

soothing resources are helpful but they are not enough. Shapiro [31] differentiated a trait change versus a state change. She defined a state change as a momentary transition versus a trait change which reflects a long-term and even permanent change. A state of change requires a continued use of coping mechanisms to maintain the change. On the other hand, a trait change does not require continued coping efforts because the client changes how she/he sees themselves, and as a result, can experience the event differently. An area for future research is to examine whether the reduction of symptoms in TF-CBT were the result of a state change since the symptoms increased again after trauma narrative; further research would examine trait change possibility with EMDR therapy since there is a continued decreasing of symptoms. Further research might include asking participants specific qualitative questions about their view of self with respect to the abuse after group treatment.

Additional finding. Six participants who completed TF-CBT group requested a subsequent EMDR group experience, which was provided for them by one of the therapists. Those participants reported that the narrative portion of TF-CBT group was triggering for them and made it difficult to achieve maximum benefit from the group. One participant reported severe stomach pains following group and, in a medical visit to the emergency room, determined that she was responding somatically to hearing another survivor's trauma narrative. 


\section{Discussion}

This study aimed to address the following research questions: 1) How effective are TF-CBT and EMDR group treatment for addressing PTSD symptoms as measured by the SPRINT instrument in Spanish speaking Latina women survivors of domestic violence? 2) Is EMDR group therapy more effective than TF-CBT group therapy in reducing PTSD symptoms in Spanish-Speaking Latina women clients who are survivors of domestic violence? Due to the exploratory nature and very limited sample in this study, only the main effect for time was statistically significant. However, the data gathered support the need for further studies to assess the differences in effectiveness of EMDR and TF-CBT in group formats since both displayed a reduction in SPRINT scores, a reduction identified as medium-to-large based on effect sizes.

The study results affirm the literature that emphasizes the benefits of group therapy interventions for survivors of domestic violence, especially when resources are limited [13]. It is also a more cost-effective treatment for Latina populations [14]. However, the researchers and clinicians experienced challenges with providing group at times when participants could attend, resulting in drop-out due to financial and transportation limitations. Agencies providing group may need to consider evening or weekend scheduling and address transportation concerns.

TF-CBT group participants reported greater symptomology at pre-test than did the EMDR group participants. However, the EMDR group saw a greater reduction in symptomology. It appears that the trauma narrative used in the TF-CBT group may contribute to higher SPRINT scores in that group at post-test. There is a possibility that group members can be re-traumatized or triggered by being exposed to others' trauma narratives sometimes noted by participants. Oneoptionis to use a TF-CBT group curriculum that provides the trauma narrative in individual or written format that does not expose other participants. A larger study with qualitative interviews might answer the question of whether the improved SPRINT scores in the EMDR groups are related to the EMDR protocol, which does not require participants to speak openly about their trauma.

\section{Limitations and Strengths of Study}

This study was an exploratory pilot study with clear need of further studies in this area. It did provide insight into possible future research questions including the role of trauma narrative in group and ways to manage the impact of other's trauma narratives on participants. A major limitation was the small sample size. While the waiting list indicated significant need and potential numbers, the sample was much smaller than anticipated. The use of group and the research design limited individual client options for therapy days and times. As a result, only 34 of the anticipated 80 participants actually enrolled in a group, and, of these 34 , only 19 completed the sessions. By the last session, some groups were 
as small as four participants. This was a significant limitation of the study. Participants dropped out for various reasons including basic needs taking priority with a few reporting an inability to tolerate treatment. One contributing limitation was lack of funding for transportation services for participants and lack of funding for child care services for children leading in some cases to participants being unable to continue in the groups. The study began with the belief that group therapy would be a way to reduce wait times for clients and address limited access to adequate service providers who speak Spanish. By clinical observation, we can state that this was accomplished since the waiting list was essentially reduced to a week or so by the offering of the last set of groups. Women who were eligible were offered groups immediately after intake with very short waits, in some cases only a week or so. At that point the waiting list included only those women who could not participate in group at the scheduled times and those who decided to wait for individual therapy slots to open. Effectively then, offering the groups did reduce the waiting list to a minimum. The study does not address the failure of many clients on the wait list to attend and/or complete the groups. This could be preference for individual group intervention or the limited time options for the groups.

Group treatment took place in the context of an agency with a long waiting list and a limited number of Spanish-speaking therapists. The Spanish speaking therapists who led the groups had been trained in both EMDR including in the Jarero group method, and in TF-CBT. One limitation of the study is that the evidence for TF-CBT is primarily for efficacy in group with children rather than adults. Studies with children include adolescents through 17 years of age, but there are not published trials with adults.

Strengths. One strength of the study is that it is a first attempt to address both a gap in the literature and a gap in service for a vulnerable population. The design of the study permitted the exploration of effectiveness of two prominent modalities in trauma intervention. The study also focused on a population that is underserved and in need of resources and a greater understanding of what treatment modalities are most effective. The therapists that lead the groups were both trained in EMDR, TF-CBT, and were Spanish-speaking Latinas. Another strength was the use of an empirically validated instrument at intake, post psychoeducation, and post intervention.

\section{Implications}

As a result of the small sample size, this study is not generalizable. It does, however, identify an important gap in the literature, important gaps in the treatment of a vulnerable population, and the importance of evaluating the effectiveness of treatment.

\section{Research}

It is important that more in-depth studies follow this exploratory study and 
greater measures be put into place to assess the efficacy of EMDR and TF-CBT in a group setting. It would also be beneficial to conduct a study with a more diverse population, larger sample size, and with multiple agencies. Another consideration that should be made in future studies is an assessment for any confounding variables that could affect SPRINT scores for participants.

Some possible questions that could be answered in future research include:

What factors affect dropout rates and how can those be managed?

Are there residual or negative effects of sharing a trauma narrative in a group setting and how can that be managed?

How many sessions are optimal to see major improvements of PTSD symptomology for each modality?

\section{Practice}

The study supports the use of group to treat an underserved population. Clearly, securing more culturally competent practitioners for underserved populations is important. Group treatment might also be an answer. Being able to utilize group formats to treat trauma could result in a reduction of waitlists. Evaluation of practice using a reliable, valid instrument allows modification of treatment approaches and the development of evidence for best practice.

\section{Political and Organizational Issues}

Other agencies may experience similar long waiting lists for therapy for Spanish-speaking Latina women. We believe the answer is access to Spanish speaking therapists with competencies in trauma therapy models. While group therapy may be an answer to therapy access, we are concerned that many did not participate in the available groups. One question is what characteristics might make some Spanish speaking Latinas better suited for group therapy than others. Agencies might begin by improving access and then evaluating therapy effectiveness including replicating this study to assess effectiveness of EMDR and TFCBT in this population. Identifying and addressing barriers to enrollment and attendance and completion are essential to providing access to treatment for vulnerable populations. Additionally, there is a clear need for more Spanish-speaking therapists for this population, particularly those who prefer individual treatment.

\section{Conclusions}

Both the EMDR and TF-CBT group showed improvement in PTSD symptoms on the SPRINT instrument. However, the EMDR group showed the greatest improvement in PTSD symptomology based on pre- and post-treatment SPRINT scores. While the limited number of subjects does not allow us to generalize, this pilot study provides some support for the efficacy of group treatment in general, with a decrease in PTSD symptomology for both groups based on means of preand post-SPRINT scores. The findings support the need for further studies to 
assess group as a treatment modality and the differences in effectiveness of EMDR and TF-CBT in group formats. If studies support the use of group formats to treat trauma, it would be beneficial for agencies to consider adopting these modalities.

This study begins to address both a gap in the literature and a gap in treatment services. Group treatment for the trauma of IVP in marginalized populations can reduce wait times and provide changes in PTSD scores. This calls for additional research. The populations with which studies have been conducted using these modalities are limited. There is a need for more and larger studies to assess group treatment effectiveness and compare these two modalities. Spanish-speaking Latinas face various forms of trauma, and Spanish speakers may feel isolated due to long wait lists and lack of service providers who can treat them. The researchers provide very preliminary evidence that it is possible to reduce wait times as well as effectively serve this population in need of improved well-being after suffering the traumatic consequences of domestic violence.

\section{Conflicts of Interest}

The authors declare no conflicts of interest regarding the publication of this paper.

\section{References}

[1] Chapman, A. and Monk, C. (2015) Domestic Violence Awareness. The American Journal of Psychiatry, 172, 944. https://doi.org/10.1176/appi.ajp.2015.15070853

[2] US Department of Justice (2015) Criminal Victimization, 2014.

[3] Fitz-Gibbon, K., Walklate, S., McCullock, J. and Maher, J.M. (2018) Intimate Partner Violence, Risk, and Security: Securing Women's Lives in a Global World. Routledge. https://doi.org/10.4324/9781315204765

[4] Regan, P.C. and Durvasula, R.S. (2015) A Brief Review of Intimate Partner Violence in the United States: Nature, Correlates, and Proposed Preventative Measures. Interpersonal, 9, 127. https://doi.org/10.5964/ijpr.v9i2.186

[5] Alvarez, C.P., Davidson, P.M., Fleming, C. and Glass, N.E. (2016) Elements of Effective Interventions for Addressing Intimate Partner Violence in Latina Women: A Systematic Review. PLoS One, 11, e0160518. https://doi.org/10.1371/journal.pone.0160518

[6] US Census (2015). https://www.census.gov/programs-surveys/acs/guidance/comparing-acs.../2015.html

[7] Black, M.C., Basile, K.C., Breiding, M.J., Smith, S.G., Walters, M.L., Merrick, M.T., Stevens, M., et al. (2011) The National Intimate Partner and Sexual Violence Survey: 2010 Summary Report. National Center for Injury Prevention and Control, Centers for Disease Control and Prevention. http://www.cdc.gov/violence prevention/pdf/nisvs_report2010-a.pdf

[8] Cuevas, C.A., Sabina, C. and Milloshi, R. (2012) Interpersonal Victimization among a National Sample of Latino Women. Violence against Women, 18, 377-403. https://doi.org/10.1177/1077801212452413

[9] McGuire, T.M., Lee, C.W. and Drummond, P.D. (2014) Potential of Eye Movement 
Desensitization and Reprocessing Therapy in the Treatment of Post-Traumatic Stress Disorder. Psychology Research and Behavior Management, 7, 273-283.

[10] Ferrari, G., Agnew-Davies, R., Bailey, J., Howard, L., Howarth, E., Peters, T.J., Feder, G.S., et al. (2016) Domestic Violence and Mental Health: A Cross-Sectional Survey of Women Seeking Help from Domestic Violence Support Services. Global Health Action, 9, 1-10. https://doi.org/10.3402/gha.v9.29890

[11] Echeburúa, E., Sarasua, B. and Zubizarreta, I. (2014) Individual Versus Individual and Group Therapy Regarding a Cognitive-Behavioral Treatment for Battered Women in a Community Setting. Journal of Interpersonal Violence, 29, 1783-1801. https://doi.org/10.1177/0886260513511703

[12] Miranda, J., Azocar, F., Organista, K.C., Muñoz, R.F. and Lieberman, A. (1996) Recruiting and Retaining Low-Income Latinos in Psychotherapy Research. Journal of Consulting and Clinical Psychology, 64, 868-874. https://doi.org/10.1037/0022-006X.64.5.868

[13] Prelli, F.A. (2014) Eye Movement Desensitization and Reprocessing (EMDR) Group Treatment Manual for Use in Women's Shelters. Unpublished Doctoral Dissertation, University of Hartford, CT.

[14] Stacciarini, J.-M.R., O'Keeffe, M. and Mathews, M. (2007) Group Therapy as Treatment for Depressed Latino Women: A Review of The Literature. Issues in Mental Health Nursing, 28, 473-488. https://doi.org/10.1080/01612840701344431

[15] O’Donnell, K., Dorsey, S., Gong, W., Ostermann, J., Whetten, R., Cohen, J.A. and Whetten, K. (2014) Treating Maladaptive Grief and Posttraumatic Stress Symptoms in Orphaned Children in Tanzania: Group-Based Trauma-Focused Cognitive-Behavioral Therapy. Journal of Traumatic Stress, 27, 664-671. https://doi.org/10.1002/jts.21970

[16] Sloan, D.M., Bovin, M.J. and Schnurr, P.P. (2012) Review of Group Treatment for PTSD. Journal of Rehabilitation Research and Development, 49, 689-702. https://doi.org/10.1682/JRRD.2011.07.0123

[17] Barron, I.G., Abdallah, G. and Smith, P. (2013) Randomized Control Trial of a CBT Trauma Recovery Program in Palestinian Schools. Journal of Loss \& Trauma, 18, 306-321. https://doi.org/10.1080/15325024.2012.688712

[18] Bisson, J. and Andrew, M. (2007) Psychological Treatment of Post-Traumatic Stress Disorder (PTSD). Cochrane Database of Systematic Reviews, 18, CD003388. https://doi.org/10.1002/14651858.CD003388.pub3

[19] Tarquinio, C., Brennstuhl, M.J., Rydberg, J.A., Schmitt, A., Moula, F., Lourel, M. and Tarquinio, P. (2012) Eye Movement Desensitization and Reprocessing (EMDR) Therapy in the Treatment of Victims of Domestic Violence: A Pilot Study. European Review of Applied Psychology, 62, 205-212. https://doi.org/10.1016/j.erap.2012.08.006

[20] Tarquinio, C., Schmitt, A., Tarquinio, P., Rydberg, J. and Spitz, E. (2012) Benefits of "Eye Movement Desensitization and Reprocessing" Psychotherapy in the Treatment of Female Victims of Intimate Partner Rape. Sexologies, 21, 60-67. https://doi.org/10.1016/j.sexol.2011.05.002

[21] Child Welfare Information Gateway (2012) US Department of Health and Human Services, Washington DC. https://www.childwelfare.gov

[22] Cohen, J.A., Mannarino, A.P. and Deblinger, E. (2012) Trauma-Focused CBT for Children and Adolescents: Treatment Applications. Guilford Publications, Inc., New York.

[23] Lowe, C. and Murray, C. (2014) Adult Service-Users' Experiences of Trauma-Focused Cognitive Behavioural Therapy. Journal of Contemporary Psychotherapy, 44, 223 
231. https://doi.org/10.1007/s10879-014-9272-1

[24] Shearing, V., Lee, D. and Clohessy, S. (2011) How Do Clients Experience Reliving as Part of Trauma-Focused Cognitive Behavioral Therapy for Post Traumatic Stress Disorder? Psychology and Psychotherapy: Theory, Research and Practice, 84, 458-475. https://doi.org/10.1111/j.2044-8341.2010.02012.x

[25] California Evidence-Based Clearinghouse for Child Welfare. http://www.cebc4cw.org/

[26] Agency for Health Care Policy and Research (2016). https://www.ncbi.nim.nih.gov/pubmed/9254637

[27] Deblinger, E., Pollio, E. and Dorsey, S. (2016) Applying Trauma-Focused Cognitive Behavioral Therapy in Group Format. Child Maltreatment, 21, 59-73. https://doi.org/10.1177/1077559515620668

[28] Deblinger, E., Stauffer, L.B. and Steer, R.A. (2001) Comparative Efficacies of Supportive and Cognitive Behavioral Group Therapies for Young Children Who Have Been Sexually Abused and Their Nonoffending Mothers. Child Maltreatment, 6, 332-343. https://doi.org/10.1177/1077559501006004006

[29] Stauffer, L.B. and Deblinger, E. (1996) Cognitive Behavioral Groups for Nonoffending Mothers and Their Young Sexually Abused Children: A Preliminary Treatment Outcome Study. Child Maltreatment, 1, 65-76.

https://doi.org/10.1177/1077559596001001007

[30] O’Callaghan, P., McMullen, J., Shannon, C., Rafferty, H. and Black, A. (2013) A Randomized Controlled Trial of Trauma-Focused Cognitive Behavioral Therapy for Sexually Exploited, War-Affected Congolese Girls. Journal of the American Academy of Child \& Adolescent Psychiatry, 52, 359-369. https://doi.org/10.1016/j.jaac.2013.01.013

[31] Shapiro, F. (2001) Eye Movement Desensitization and Reprocessing (EMDR). 2nd Edition, Guilford Press, New York.

[32] Acarturk, C., Konuk, E., Cetinkaya, M., Senay, I., Sijbrandij, M., Gulen, B. and Cuijpers, P. (2016) The Efficacy of Eye Movement Desensitization and Reprocessing for Post-Traumatic Stress Disorder and Depression Among Syrian Refugees: Results of a Randomized Controlled Trial. Psychological Medicine, 46, 2583-2593. https://doi.org/10.1017/S0033291716001070

[33] Chen, Y.-R., et al. (2014) Efficacy of Eye-Movement Desensitization and Reprocessing for Patients with Posttraumatic-Stress Disorder: A Meta-Analysis of Randomized Controlled Trials. PLos ONE, 9, e103676. https://doi.org/10.1371/journal.pone.0103676

[34] de Bont, P.A.J.M., van Minnen, A. and de Jongh, A. (2013) Treating PTSD in Patients with Psychosis: A Within-Group Controlled Feasibility Study Examining the Efficacy and Safety of Evidence-Based PE and EMDR Protocols. Behavior Therapy, 44, 717-730. https://doi.org/10.1016/j.beth.2013.07.002

[35] Ringel, S. (2014) An Integrative Model in Trauma Treatment: Utilizing Eye Movement Desensitization and Reprocessing and a Relational Approach with Adult Survivors of Sexual Abuse. Psychoanalytic Psychology, 31, 134-144. https://doi.org/10.1037/a0030044

[36] Vaishnavi, S., Payne, V., Connor, K. and Davidson, J.R.T. (2006) A Comparison of the SPRINT and CAPS Assessment Scales for Posttraumatic Stress Disorder. Depression and Anxiety, 23, 437-440. https://doi.org/10.1002/da.20202

[37] Jarero, I. and Artigas, L. (2010) The EMDR Integrative Group Protocol: Application with Adults during Ongoing Geopolitical Crisis. Journal of EMDR Practice and Re- 
search, 4, 148-155. https://doi.org/10.1891/1933-3196.4.4.148

[38] Jarero, I., Artigas, L. and Hartung, J. (2006) EMDR Integrative Group Treatment Protocol: A Post Disaster Trauma Intervention for Children and Ddults. Traumatology, 12, 121-129. https://doi.org/10.1177/1534765606294561

[39] Jarero, I., Artigas, L., Uribe, S., Garcia, L.E., Cavazos, M.A. and Givaudan, M. (2015) Pilot Research Study on the Provision of the Eye Movement Desensitization and Reprocessing Integrative Group Treatment Protocol with Female Cancer Patients. Journal of EMDR Practice and Research, 9, 98-105. https://doi.org/10.1891/1933-3196.9.2.98

[40] Connor, K.M. and Davidson, J.R.T. (2001) SPRINT: A Brief Global Assessment of Post-Traumatic Stress Disorder. International Clinical Psychopharmacology, 16, 279-284. https://doi.org/10.1097/00004850-200109000-00005

[41] Leiva-Bianchi, M.C. and Gallardo, I. (2013) Validation of the Short Posttraumatic Stress Disorder Rating Interview (SPRINT-E) in a Sample of People Affected by F-27 Chilean Earthquake and Tsunami. Anales De Psicología, 29, 328-334. 